\title{
Prostate-specific antigen and unwarranted use of costly investigations in medical practice
}

\author{
Anuruddha M Abeygunasekera \\ Urological Surgeon, Teaching Hospital, Karapitiya, Galle.
}

Unnecessary testing is becoming increasingly common in medical practice and consumer demand for certain type of investigations has escalated. Such testing is expensive and diverts patient and clinicians' time from addressing more genuine needs. In addition, these can provide unwarranted reassurance or cause unnecessary anxiety and can lead to further interventions that may carry risks of significant morbidity and even mortality. Unnecessary testing has been promoted by both well-intentioned medical practitioners and outright quacks for centuries. Due to lack of health literacy and/or powerful need for reassurance, many individuals are willing to pay, sometimes exorbitant amounts for screening tests that lack scientific merit. Some professionals and institutes prey on fear of serious illnesses and on an individual's natural desire to detect health problems early in hopes of achieving a cure.

Testing serum prostate-specific antigen (PSA) is a classic example of this trend. In the past three decades since its discovery, PSA has been recognised as the most effective tumour marker for prostate cancer. It has unquestionably played an important role in the diagnosis and clinical management of this disease [1]. Despite the success of PSA as a tumour marker both clinical and analytical limitations of PSA remain. Hence, interpretation of PSA results and subsequent decision making is a complex process which requires critical analysis and wise clinical judgment.

To begin with, there is no consensus on the upper limit of the normal range for serum PSA. Some believe it should be $2.6 \mathrm{ng} / \mathrm{mL}$ and not $4.0 \mathrm{ng} / \mathrm{mL}$ in order to avoid missing early prostatic malignancies [2]. Inter-racial difference in the normal level and inter-lab variations of PSA estimations are well known [3]. Differences may persist even among assays from the same manufacturer that have the same antibodies but different assay formats [4]. With most of the laboratories in Sri Lanka lacking any form of standardisation and quality assurance, potential discrepancies in PSA levels due to analytical errors should be borne in mind. Lack of data regarding the norms for PSA levels in Sri Lankan men compounds the issue further. It has been shown that even the effect of age on PSA is different in different populations [5].

Although PSA is considered an effective tumour marker and is organ specific, there is considerable overlap in PSA concentrations in men with prostatic carcinoma and men with benign prostatic diseases. Even attempts to improve the diagnostic accuracy of PSA including age-specific PSA, free PSA, PSA density, PSA velocity and the molecular forms of PSA have failed to improve the specificity any further significantly despite the initial enthusiasm. Many other factors have been incriminated to affect the PSA levels. These include acute bacterial prostatitis, acute urinary retention, recent ejaculation, prostate massage, prostate biopsy and transurethral resection of the prostate [6]. In fact false positives are so common with PSA some recommend a second PSA assay in six weeks before proceeding for a biopsy after an initially high PSA [7].

Although PSA is the best tumour marker currently available for detecting prostatic carcinoma, its suitability as a screening tool remains controversial. Therefore, even the developed countries do not recommend screening programmes using PSA to detect early prostatic carcinoma [8]. Although the experts continue to argue about the evidence on 
screening, the public and many 'non-urological' medical personnel have come to different conclusions. Epidemiological or clinical evidence may play a small part in the public's demand for screening for prostate cancer by PSA testing. Instead the irresistible logic of finding the cancer early, the drive to avoid regretting later their decision not to have the test and the right to obtain information about oneself by testing may all be more important arguments. These lay arguments for PSA testing have their own logic and validity. In this era of litigation and defensive medicine the experts may also be inclined to be swept away by these demands. But the consequences of screening can be far reaching than we may imagine. Screening is costly, particularly for a developing country like Sri Lanka where health care funds are limited. Furthermore, screening is a process of changing the identities; it is not a trivial matter. It has profound health, social, psychological and economic consequences [9]. Screening therefore raises important ethical problems. Hence, use of PSA as a screening tool must be based on sound research and good governance.

Many clinicians in USA promote aggressive testing for serum PSA with the hope of identifying patients with early and potentially more curable prostate cancer [10]. However, the impact of identifying prostate cancers early using PSA on longterm survival is still a contentious issue [11]. Many cite improvements in improved survival rates. PSA testing has advanced the time of diagnosis of prostate cancer by as much as 5-10 years [12]. This lead time bias alone will appear to improve survival rates dramatically. Clinical outcomes can be influenced by another factor Gleason score shift [13,14]. Although the Gleason scoring system itself has not changed its application has. Clinicians treating contemporary populations with newly diagnosed prostate cancer in USA rarely encounter men with low Gleason scores, whereas two decades ago low Gleason scores were very common [15]. One possibility for this change is that pathologists are more hesitant to assign low Gleason scores to contemporary prostatic biopsy specimens because these scores are frequently upgraded after review of the main surgical specimen of radical prostatectomy. Early days when radical surgery was not done, only the low Gleason score of the biopsy was available for analysis. If this explanation is correct the resulting shift in Gleason scores would lead to apparent improvements in survival when, in fact such improvements have not occurred.

Many clinicians in this country use serum PSA for 'case-finding' prostate cancer within those patients presenting with acute urinary retention (AUR). AUR leads to a temporary elevation of serum PSA levels. Although the precise mechanism is unclear, it is believed to be due to prostatic infarction [16]. Prostatic biopsies are done unnecessarily after seeing high PSA values obtained on patients admitted with AUR. This is further compounded by the fact that it occurs in a relatively elderly group who may have significant co-morbidity. This may lead to unnecessary complications with significant health, social, psychological and economic implications. Serum PSA estimation in the private sector will cost about Rs. 3,000/=. According to the Central Bank figures, the average annual income of a Sri Lankan is Rs. $4,326 /=[17]$. It is not uncommon for some clinicians to request serum PSA estimation even without assessing the prostate clinically by digital rectal examination.

The use of clinically unjustifiable tests erodes the scientific basis of medical practice and sends a wrong message to trainees and patients about when and why to use diagnostic studies. Such use also runs counter to physicians ethical obligations to contribute to the responsible stewardship of health care resources. Without adequate education and knowledge, clinicians may lack the courage to refuse unwarranted testing. Commercial interests worsen the situation.

Improved science $\&$ health education, scientific integrity of individuals \& institutes and better communication between patients \& healthcare providers would help to increased use of appropriate, less harmful and less costly investigations which will enhance health outcomes. 


\section{References}

1. Sokoll LJ, Chan DW. Prostate-specific antigen. Its discovery and biochemical characteristics. Urological Clinics of North America 1997; 24: 253-9.

2. Kravchick S, Peled R, Dorfman D, Agulansky L, Cytron S. Predictive criteria for prostate cancer detection in men with serum prostate-specific antigen concentration of 2.0 to $4.0 \mathrm{ng} / \mathrm{mL}$. Urology 2005; 66: 542-6.

3. Morgan TO, Jacobsen SJ, McCarthyn F, Jacobsen DJ, McLeod DJ. Age-specific reference ranges for serum Prostate-specific antigen in black men. New England Journal of Medicine 1996; 335: 304-10.

4. Chan DW, Sokoll LJ. WHO first International Standards for Prostate-specific Antigen: The beginning of the end for assay discrepancies? Clinical Chemistry 2000; 46: 1291-2.

5. Kobayashi T, Kinoshita H, Nishizawa K, Mitsumori K, Ogawa O. Age-associated increase of PSA in a high level of men visiting urological clinics. International Journal of Urology 2005; 12: 733-8.

6. Oesterling JE. Age-specific reference ranges for serum PSA. New England Journal of Medicine 1996: 335 : 345-6.

7. Eastham JA, Riedel E, Scardino PT, Shike M, Fleisher M. Variation of serum prostate-specific antigen levels: an evaluation of year to year fluctuation. Journal of the American Medical Association 2003; 289: 2695-700.

8. Frankel S, Smith GD, Donovan J, Neal D. Screening for prostate cancer. Lancet 2003;361: 1122-7.

9. Thornton H. Consequences of screening. Lancet 2000; 356: 1033.
10. Han M, Partin AW, Piantadosi S, Epstein JI, Walsh PC. Era-specific biochemical recurrence-free survival following radical prostatectomy for clinically localized prostate cancer. Journal of Urology 2001; 166: 416-9.

11. Whalen P. The case against radical prostatectomy. Annals of the Royal College of Surgeons of England 2005; 87: 159-62.

12. DraismaG, Boer R, Otto SJ, Damhuis RA, Schroder FH et al. Lead times and over-detection due to prostatespecific antigen screening: estimates from the European randomized study of screening for prostate cancer. Journal of the National Cancer Institute 2003; 95; 868-78.

13. Welch HG, Schwatz LM, Woloshin S. Are increasing 5-year survival rates evidence of success against cancer? Journal of the American Medical association 2000; 283: 2975-8.

14. Smith EB, Frierson HF, Mills SE, Boyd JC, Theodorescu D. Gleason scores of prostatic biopsy and radical prostatectomy specimens over the past 10 years. Cancer 2002; 94: 2282-7.

15. Jhaveri FM, Klein EA, Kupleian PA, Zipper C, Levin HS. Declining rates of extracapsular extension after radical prostatectomy: evidence for continued migration. Journal of Clinical Oncology 1999; 17: 3167-72.

16. Hagood PG, Parra RO, Rauscher JA Non-traumatic elevation of prostate-specific antigen following cardiac surgery and extracorporeal cardiopulmonary bypass. Journal of Urology 1994; 152: 2043-5.

17. Annual Report: Central Bank of Sri Lanka 2003-2004. 